\title{
Epidemiological study of pathogens isolated from blood in Liguria during 20 II |
}

Ramona Barbieri', Erika Coppo', Luigi Carlo Bottaro², Paolo Piazzai' ${ }^{2}$ Orietta Illiberi², Pier Andrea Dusi ${ }^{3}$, Rita Revello $^{3}$, David Usiglio4, Marco Mori ${ }^{4}$, Silvia Reali $^{5}$, Gian Luigi Devoto ${ }^{5}$, Luisa Santoriello ${ }^{6}$, Agostina Ronca $^{6}$, Anna Marchese', Eugenio A. Debbia'

I Sezione di Microbiologia DISC, University of Genova

2 International Evangelical Hospital, Genova-Voltri;

3 ASL I Imperiese, Sanremo Hospital (Imperia);

4 Ente Ospedaliero "Galliera", Genova;

5 ASL4 Chiavarese, Genova;

6 Santa Corona Hospital, Pietra Ligure (Savona)

Key words: Blood culture, Drug resistance, Bacteraemia.

Studio epidemiologico di patogeni isolati dal sangue in Liguria (marzo-maggio 20 I I)

\section{SUMMARY}

Objectives. An epidemiological study addressed to identify the most represented pathogens isolated from blood and to evaluate their antibiotic susceptibility patterns, was conducted.

Methods. Five clinical microbiology laboratories, homogenously distributed in Liguria, were required to collected all consecutive non-duplicates strains isolated from blood cultures during March $201 \mathrm{I}$ to May $201 \mathrm{I}$. the strains were sent to the reference laboratory (Section of Microbiology, DISC, University of Genoa, Italy).

Results. A total of 159 microorganisms were enrolled, including 81 Gram positive, $69 \mathrm{Gram}$ negative and 9 fungi. The most represented pathogens were: Escherichia coli (35), Staphylococcus aureus (26), S. epidermidis (20), S. hominis (10).

Samples were collected mainly from medicine (59 isolates). Among the staphylococci, the most active molecules were: vancomycin (100\% of susceptible strains), teicoplanin ( $93.4 \%)$, trimethoprim-sulfamethoxazole $(83.8 \%)$ and tobramycin (61.6\%). Enterococci showed rates of resistance to vancomycin of $25 \%$. Enterobacteriaceae exhibited resistance to ampicillin (76.9\%), ceftriaxone $(44.4 \%)$, ciprofloxacin (43.3\%), trimethoprim-sulfamethoxazole (36.6\%) and ceftazidime (32.2\%).

Conclusions. The data show a higher incidence of Gram positive (5I\%) in comparison to Gram negative (43.4\%). Gram-positive strains showed a high resistance level to fluoroquinolones (92.3\%) while Gram-negative resulted resistant to ceftriaxone $(44.4 \%)$ and fluoroquinolone $(43.3 \%)$.

\section{INTRODUCTION}

There are two mechanisms by which bacteria can enter the blood: drainage from the primary focus of infection via the lymphatic to the vascular system, or direct entry from needles or other contaminated intravascular devices such as catheters or graftmaterial (4).

Catheter-related bloodstream infections are among the most common nosocomial infections in critical patients and are associated with significant morbidity and mortality $(2,3,6)$.

The presence of a bloodstream infection represents either the failure of an individual's host defenses to localize an infection at its primary site or the failure of a physician to remove, drain, or otherwise sterilize that focus. Finally, host defenses respond promptly to a sudden influx of microorganisms, particularly by efficient phago- cytosis by macrophages or the mononuclear phagocytic system that helps clear the blood within minutes to hours. Clearance may be less efficient when microorganisms are encapsulated, or it may be enhanced if the host has antibodies specific for the infecting organism. Clearance of the bloodstream is not always successful.

Examples of this problem are bloodstream infections associated with intravascular foci and endovascular infections and episodes that occur in individuals whose host defense mechanisms either are too impaired to respond efficiently or are simply overwhelmed.

For that reason, the presence of living microorganisms in blood is of substantial clinical importance; it is an indicator of disseminated infection and, as such, generally indicates a poorer prognosis than that associated with localized disease.

\section{Corresponding author: Ramona Barbieri}

Sezione di Microbiologia, C.A. Romanzi; Facoltà di Medicina e Chirurgia-DISC

Largo Rosanna Benzi, I0 - I6I32 Genova - Tel.: 0103538998 - Fax: 010353765 I

E-mail: ramona.barbieri@unige.it 
According to clinical patterns of bacteremia, it may also be useful to categorize bloodstream infection as transient, intermittent, or continuous (4). Transient bacteremia, lasting minutes to hours, is the most common and occurs after manipulation of infected tissues (e.g., abscesses); during certain surgical procedures; when procedures are undertaken that involve contaminated or colonized mucosal surfaces (e.g., gastrointestinal endoscopy); and, predictably, at the onset of acute bacterial infections such as pneumonia, meningitis, and complicated urinary infections.

Intermittent bacteremia is that which occurs, clears, and then recurs in the same patient due to the same microorganism.

Classically, this type of bacteremia is associated with undrained closed space infections, such as intra-abdominal abscesses. Continuous bacteremia is characteristic of infective endocarditis as well as other endovascular infections such as arterial graft infections, and suppurative thrombophlebitis associated with intravenous line infections commonly seen in critically ill patients. Bloodstream infections may also be categorized as monomicrobial or polymicrobial depending on the number of microorganisms isolated during a single bacteremic

episode. Several techniques are available to assist the clinician and microbiologist in interpreting the clinical importance of a positive blood culture.

The categorical decision to consider the bloodstream infection as true infection or a contaminant should take into account, at least: the patient's clinical history, physical findings, body temperature at the time of the blood culture,

leukocyte count and differential cell counts, the identity of microorganism isolated and the result of cultures of specimens from other sites.

Indeed, the type of microorganism isolated may have some predictive value: common blood isolates that always or nearly always $(>90 \%)$ represent true infection include $S$. aureus, E. coli and other members of the Enterobacteriaceae, Pseudomonas aeruginosa, Streptococcus pneumoniae, and Candida albicans.

Inappropriate empirical antimicrobial treatment was considered as the occurrence of bloodstream infection that was not effectively treated at the time when the causative microorganism and its antibiotic susceptibility were known, including the absence of antimicrobial agents directed at a specific class of microorganisms and the administration of an antimicrobial agent to which the microorganism responsible for the infection was resistant (5).

For all reasons we aimed to identify the most represented pathogens isolated from blood and to evaluate their antibiotic susceptibility patterns.

\section{MATERIAL AND METHODS \\ Bacterial isolates}

This study was conducted during March 2011 to May 2011 with the collaboration of five clinical microbiology laboratories homogeneously distributed in the Ligurian area. The enrolled laboratories were: International Evangelical Hospital San Carlo, Genoa-Voltri; ASL 1 Sanremo Hospital (Imperia); Ente Ospedaliero "Galliera", Genoa; ASL 4 Chiavarese, Genoa; Santa Corona Hospital, Pietra Ligure (Savona) Overall, 159 microorganisms were collected and sent to the reference laboratory (Section of Microbiology, DISC, University of Genoa). All consecutive nonduplicates strains isolated from blood cultures were studied.

Antimicrobial Susceptibility tests. The Minimum inhibitory concentrations of penicillin, ampicillin, piperacillin-tazobactam, ceftriaxone, ceftazidime, cefotaxime, cefoxitin, cefepime, imipenem, meropenem, amikacin, gentamicin, ciprofloxacin, trimethoprim-sulfamethoxazole, teicoplanin, vancomycin, azitromycin, eritromicin/claritromicin, amikacin, amoxicillin/clavulanate, aztreonam, chloramphenicol, tetracyclin were determined by the broth microdilution method, according to the Clinical Laboratory Standards Institute (CLSI) recommendations (1). Escherichia coli ATCC 25922, E. coli ATCC 35218, Pseudomonas aeruginosa ATCC 27853 and Staphylococcus aureus ATCC 25923 were used as quality controls strains. The results were reported as susceptible resistant in accordance to CLSI raccomandation and compared with those obtained by the original laboratory.

\section{RESULTS}

A total of 159 microorganisms were found, including 81 Gram positive, 69 Gram negative and 9 fungi.

E. coli stands out among both Gram-negative absolute the total number of isolates. In the Table 1 was summarised the complete list and the distribution of the pathogens collected in this study. The most represented pathogens were: E. coli (35), S. aureus (26), S. epidermidis (20), S. hominis (10).

The pathogens were obtained mainly from patients hospitalized in general medicine wards (59 isolates), Intensive Care Units (17) and surgery (15).

Among the Gram negative strains, $87 \%$ of these were Enterobacteriaceae and 13\% were non Enterobacteriaceae. The majority $(77.7 \%)$ of 
Gram positive strains was represented by Staphylococci, $12.3 \%$ consisted of Streptococci and 10\% were Enterococci.

The antibiotics susceptibility patterns of the strains collected were showed in Table 2.

Among staphylococci, the most active molecules were: vancomycin (100\% of susceptible strains), teicoplanin $(93.4 \%)$, trimethoprim-sulfamethoxazole $(83.8 \%)$ and tobramycin $(61.6 \%)$.

As regards the OXA-R Staphylococci (40 isolates), the most active molecules were: vancomycin $(100 \%$ of susceptible strains), teicoplanin $(89.7 \%)$, trimethoprim-sulfamethoxazole $(77.5 \%)$ and tobramycin $(72.5 \%)$.

Enterococci showed rates of resistance to vancomycin of $25 \%$.

Enterobacteriaceae exhibited resistance to ampicillin $(76.9 \%)$, ceftriaxone $(44.4 \%)$, ciprofloxacin (43.3\%), trimethoprim-sulfamethoxazole $(36.6 \%)$ and ceftazidime $(32.2 \%)$.

The Gram negative non- Enterobacteriaceae showed rates of resistance of $83.4 \%$ to piperacillin/tazobactam, of $50 \%$ to amikacin and imipenem, of $37.5 \%$ to ciprofloxacin, of $33.4 \%$ to trimethoprim-sulfamethoxazole and ceftazidime. Table 3 showed the number of Staphylococci have shown phenotypes of resistance to one or more classes of antibiotics. S. epidermidis was the species that more than others has showed phenotypes of multiresistance to antibiotics.

S. aureus showed a oxacillin-resistance of $49.1 \%$, while the multiresistance to antibiotics has been observed in more than one one third of isolates insensitive to oxacillin. S. hominis and $\mathrm{S}$. haemolyticus showed high level of resistance to the great majority of the antibiotics. Among Gram-negative strains (Table 4), E. coli manifested high level of multidrug resistance in $51.5 \%$ of tested isolates.

The other Gram-negative strains although they were isolated a small number showed high levels of multidrug resistance.

In few cases the result were not in accordance with those obtained by the original laboratory. These isolates show susceptibility to 2 or 3 classes of antibiotics in comparison to the reported by the original laboratory.

\section{CONCLUSIONS}

The present study detected a high incidence of Gram positive (51\%) in comparison to Gram negative $(43.4 \%)$. E. coli is the most isolated pathogen followed by $S$. aureus, $S$. epidermidis and $S$. hominis. The high incidence of oxacillinoresistance in Staphylococci $(63.5 \%)$ was confirmed. Against Enterobacteriaceae rates of resistance were observed in excess of $20 \%$ for all drugs tested excepted imipenem $(10.5 \%)$ and amikacin $(8.5 \%)$.

Glycopeptides were the most active molecules against Staphylococci and Enterococci.

Piperacillin-tazobactam was the most effective molecule against Gram-negative strains.

The purpose of this study is to emphasize the importance of knowing the species most isolated from the blood and their resistance to antibiotics because, in absence of microbiological data, the therapy is mainly empirical.

Table I. Distribution of the strains collected.

\begin{tabular}{|c|c|c|c|c|c|}
\hline $\begin{array}{l}\text { Gram negative } \\
\text { strains }\end{array}$ & $\begin{array}{c}\text { N. of } \\
\text { isolated }\end{array}$ & $\begin{array}{c}\text { Gram positive } \\
\text { strains }\end{array}$ & $\begin{array}{c}\text { N. of } \\
\text { isolated }\end{array}$ & Fungi & $\begin{array}{c}\text { N. of } \\
\text { isolated }\end{array}$ \\
\hline E. coli & 35 & S. aureus & 26 & C. albicans & 4 \\
\hline K. pneumoniae & 7 & S. epidermidis & 20 & C. parapsilosis & 4 \\
\hline P. aeruginosa & 5 & S. hominis & 10 & C. tropicalis & 1 \\
\hline P. mirabilis & 5 & S. pneumoniae & 6 & & \\
\hline E. cloacae & 4 & S. haemolyticus & 4 & & \\
\hline K. oxytoca & 3 & E. faecalis & 4 & & \\
\hline A. baumannii & 2 & E. faecium & 4 & & \\
\hline B. vesicularis & $\mathrm{I}$ & S. agalactiae & 2 & & \\
\hline C. freundii & $\mathrm{I}$ & S. lugdunensis & 1 & & \\
\hline E. aerogenes & 1 & S. capitis & 1 & & \\
\hline M. morganii & $\mathrm{I}$ & S. dysagalactiae & 1 & & \\
\hline P. putida & $\mathrm{I}$ & S. pyogenes & 1 & & \\
\hline P. stuartii & $\mathrm{I}$ & S. schleiferi & $\mathrm{I}$ & & \\
\hline S. maltophilia & $\mathrm{I}$ & & & & \\
\hline S. typhimurium & I & & & & \\
\hline Total & 69 & Total & 81 & Total & 9 \\
\hline
\end{tabular}


Table 2. Percentages of resistance to major classes of antibiotics.

\begin{tabular}{|c|c|c|c|c|c|c|c|c|c|c|c|c|c|c|c|c|c|}
\hline \multirow{2}{*}{$\begin{array}{c}\text { Strains } \\
\text { (Number of strains } \\
\text { collected) }\end{array}$} & \multicolumn{17}{|c|}{ (Number of strains tested) } \\
\hline & PEN & OX & AMP & TZP & $\begin{array}{l}C R \\
O\end{array}$ & $\begin{array}{c}\text { CA } \\
\mathrm{Z}\end{array}$ & FOX & FEP & IMI & AMK & GEN & $\begin{array}{l}\text { CIPI } \\
\text { LEV }\end{array}$ & SXT & TEC & VAN & AZI & ERY \\
\hline Staphylococci (63) & & $\begin{array}{l}65.6 \\
(61)\end{array}$ & & & & & & & & & $\begin{array}{l}41.9 \\
(62)\end{array}$ & $\begin{array}{l}57.1 \\
(21)\end{array}$ & $\begin{array}{l}16.1 \\
(62)\end{array}$ & $\begin{array}{l}6.5 \\
(61)\end{array}$ & $\begin{array}{l}0.0 \\
(62)\end{array}$ & $\begin{array}{l}40 \\
(5)\end{array}$ & $\begin{array}{l}62.3 \\
(61)\end{array}$ \\
\hline $\begin{array}{c}\text { Staphylococci Oxa- } \\
R(40)\end{array}$ & $\begin{array}{l}100 \\
(40)\end{array}$ & & & & & & & & & & $\begin{array}{l}57.5 \\
(40)\end{array}$ & $\begin{array}{l}92.3 \\
(13)\end{array}$ & $\begin{array}{l}22.5 \\
(40)\end{array}$ & $\begin{array}{l}10.3 \\
(39)\end{array}$ & $\begin{array}{l}0.0 \\
(40)\end{array}$ & & $\begin{array}{c}85 \\
(40)\end{array}$ \\
\hline $\begin{array}{c}\text { Enterobacteriaceae } \\
(60)\end{array}$ & & & $\begin{array}{l}76.9 \\
(52)\end{array}$ & $\begin{array}{l}23.9 \\
(46) \\
\end{array}$ & $\begin{array}{l}44.4 \\
(18)\end{array}$ & $\begin{array}{l}32.2 \\
(59)\end{array}$ & $\begin{array}{l}21.4 \\
(14) \\
\end{array}$ & $\begin{array}{c}30 \\
(60)\end{array}$ & $\begin{array}{l}10.5 \\
(57) \\
\end{array}$ & $\begin{array}{r}8.5 \\
(59)\end{array}$ & & $\begin{array}{l}43.3 \\
(60) \\
\end{array}$ & $\begin{array}{l}36.6 \\
(60) \\
\end{array}$ & & & & \\
\hline $\begin{array}{c}\text { Gram negative non } \\
\text { Enterobacteriaceae } \\
(9)\end{array}$ & & & & $\begin{array}{l}16.6 \\
(6)\end{array}$ & $\begin{array}{l}100 \\
(5)\end{array}$ & $\begin{array}{c}66.6 \\
(6)\end{array}$ & $\begin{array}{l}100 \\
(4)\end{array}$ & $\begin{array}{c}66.6 \\
(6)\end{array}$ & $\begin{array}{l}50 \\
(8)\end{array}$ & $\begin{array}{l}50 \\
(8)\end{array}$ & & $\begin{array}{c}62.5 \\
(8)\end{array}$ & $\begin{array}{c}66.6 \\
(6)\end{array}$ & & & & \\
\hline
\end{tabular}

Pen, penicillin; amp, ampicillin; tzp, piperacillin-tazobactam; cro, ceftriaxone; caz, ceftazidime; fox, cefoxitin; fep, cefepime; imi, imipenem; amk, amikacin; gen, gentamicin; cip, ciprofloxacin; lev: levofloxacin; sxt, trimethoprim-sulfamethoxazole; tec, teicoplanin; van, vancomycin, azi azithromycin; ery, erythromycin/clarithromycin.

Table 3. Distribution of resistance to oxacillin (OXA) alone and in combination with other classes of antibiotics in staphylococci.

\begin{tabular}{|c|c|c|c|c|c|c|}
\hline Strains & $\begin{array}{c}\text { N. of } \\
\text { isolates }\end{array}$ & $\begin{array}{c}\text { OXA-R } \\
\text { (N.) }\end{array}$ & $\%$ & $\begin{array}{c}\text { OXA-R } \\
+ \text { IR }\end{array}$ & $\begin{array}{c}\text { OXA-R } \\
+2 R\end{array}$ & $\begin{array}{c}\text { OXA-R } \\
\geq 3 R\end{array}$ \\
\hline S. aureus & 26 & 15 & 57.7 & I & 3 & II \\
\hline S. epidermidis & 20 & 15 & 75 & 2 & 2 & II \\
\hline S. hominis & 10 & 7 & 70 & I & $\mathrm{I}$ & 6 \\
\hline S. haemolyticus & 4 & 3 & 75 & I & I & 3 \\
\hline Other CNS* & 3 & 1 & 1 & 1 & 1 & 1 \\
\hline & 63 & 40 & 63.5 & 3 & 6 & 31 \\
\hline
\end{tabular}

Table 4. Distribution of single and multiple antibiotic resistance in Gram-negative strains.

\begin{tabular}{|c|c|c|c|c|c|}
\hline Strains & N. of isolates & $\mathbf{S}$ & I R & $2 \mathbf{R}$ & $3 \mathbf{R}$ \\
\hline E. coli & 35 & 8 & 4 & 5 & 18 \\
\hline K.pneumoniae & 7 & 1 & 1 & 1 & 7 \\
\hline P. aeruginosa & 5 & 1 & $\mathrm{I}$ & 1 & 4 \\
\hline P. mirabilis & 5 & 1 & $\mathrm{I}$ & 1 & 4 \\
\hline E. cloacae & 4 & 1 & 3 & 1 & 1 \\
\hline K. oxytoca & 3 & 2 & $\mathrm{I}$ & 1 & 1 \\
\hline A. baumannii & 2 & $\mathrm{I}$ & 1 & 1 & $\mathrm{I}$ \\
\hline Other* & 7 & 1 & $\mathrm{I}$ & 2 & 4 \\
\hline
\end{tabular}

* B. vesicularis (I), C. freundii (I), E. aerogenes (I), M. morganii (I), P. putida (I), S. typhimurium (I).

\section{REFERENCES}

1. Clinical and Laboratory Standards Institute (CLSI). Performance Standards for Antimicrobial Susceptibility Testing: Twenty-second Informational Supplement. 2011; M100-S21. CLSI, Wayne, PA, USA.

2. Edgeworth JD, Treacher DF, Eykyn SJ. A 25-year study of nosocomial bacteraemia in an adult intensive care unit. Crit Care Med. 1999; 27: 1421-8.

3. Pittet D, Tarara D, Wenzel RP. Nosocomial bloodstream infection in critically ill patients: excess length of stay, extra costs, and attributable mortality. JAMA. 1994; 271: 1598-601.

4. Reimer LG, Wilson ML, Weinstein MP. Update on detection of bacteremia and fungemia. Clin Microbiol Rev 1997; 10: 444-65.

5. Trilla A, Gatell JM, Mensa J, et al. Risk factors for nosocomial bacteremia in a large Spanish teaching hospital: A case-control study. Infect Control Hosp Epidemiol 1991; 12: 150-6.

6. Wenzel RP, Edmond MB. The impact of hospitalacquired bloodstream infections. Emerg Infect Dis. 2001; 7: 174-7. 